Draft Version November 7, 2018

Preprint typeset using $\mathrm{IAT}_{\mathrm{E}} \mathrm{X}$ style emulateapj v. 5/2/11

\title{
DEEP CHANDRA OBSERVATIONS OF THE COMPACT STARBURST GALAXY HENIZE 2-10: X-RAYS FROM THE MASSIVE BLACK HOLE
}

\author{
Amy E. REINES ${ }^{1}$ \\ National Optical Astronomy Observatory, 950 North Cherry Avenue, Tucson, AZ 85719, USA and \\ Department of Astronomy, University of Michigan, 1085 South University Avenue, Ann Arbor, MI 48109, USA \\ MARK T. REYNOLDS \\ Department of Astronomy, University of Michigan, 1085 South University Avenue, Ann Arbor, MI 48109, USA \\ JoN M. MilleR \\ Department of Astronomy, University of Michigan, 1085 South University Avenue, Ann Arbor, MI 48109, USA \\ Gregory R. SivakofF \\ Department of Physics, University of Alberta, CCIS 4-181, Edmonton AB T6G 2E1, Canada \\ Jenny E. GReene \\ Department of Astrophysical Sciences, Princeton University, Princeton, NJ 08544, USA \\ RYAN C. HICKOX \\ Department of Physics and Astronomy, Dartmouth College, 6127 Wilder Laboratory, Hanover, NH 03755, USA \\ AND \\ KELSEY E. JOHNSON \\ Department of Astronomy, University of Virginia, P.O. Box 400325, Charlottesville, VA 22904-4325, USA \\ Draft version November 7, 2018
}

\begin{abstract}
We present follow-up X-ray observations of the candidate massive black hole (BH) in the nucleus of the low-mass, compact starburst galaxy Henize 2-10. Using new high-resolution observations from the Chandra X-ray Observatory totaling 200 ks in duration, as well as archival Chandra observations from 2001, we demonstrate the presence of a previously unidentified X-ray point source that is spatially coincident with the known nuclear radio source in Henize 2-10 (i.e., the massive BH). We show that the hard X-ray emission previously identified in the 2001 observation is dominated by a source that is distinct from the nucleus, with the properties expected for a high-mass X-ray binary. The X-ray luminosity of the nuclear source suggests the massive BH is radiating significantly below its Eddington limit $\left(\sim 10^{-6} L_{\mathrm{Edd}}\right)$, and the soft spectrum resembles other weakly accreting massive BHs including Sagittarius A*. Analysis of the X-ray light curve of the nucleus reveals the tentative detection of a $\sim 9$-hour periodicity, although additional observations are required to confirm this result. Our study highlights the need for sensitive high-resolution X-ray observations to probe low-level accretion, which is the dominant mode of $\mathrm{BH}$ activity throughout the Universe.
\end{abstract}

Subject headings: accretion, accretion disks — galaxies: active — galaxies: dwarf — galaxies: nuclei - X-rays: general

\section{INTRODUCTION}

Henize 2-10 is a remarkable compact starburst galaxy, hosting an abundance of young "super star clusters" (e.g., Johnson et al. 2000) and a candidate low-luminosity active galactic nucleus (AGN) (Reines et al. 2011). The discovery of an AGN in Henize 2-10 provides an excellent opportunity to study $\mathrm{BH}$ accretion and star formation in a nearby $(\sim 9 \mathrm{Mpc})$, low-mass $\left(\lesssim 10^{10} M_{\odot}\right.$; Nguyen et al. 2014), gas-rich galaxy, as well as the potential formation of a nuclear star cluster around a preexisting massive BH (Nguyen et al. 2014: Arca-Sedda et al. 2015). More-

reines@noao.edu

${ }^{1}$ Hubble Fellow over, this finding has helped spark a number of recent searches for AGNs in dwarf galaxies (e.g., Reines et al. 2013, 2014, Baldassare et al. 2015; Lemons et al. 2015, Baldassare et al.| 2016: Hainline et al. 2016), ultimately leading to the realization that massive $\mathrm{BHs}$ in dwarfs are much more common than previously thought (for a review, see Reines \& Comastri 2016).

The evidence for a massive $\mathrm{BH}$ in Henize 2-10 comes from a wealth of multi-wavelength data (Reines et al. 2011), including Very Large Array (VLA) radio observations that reveal an unresolved non-thermal nuclear point source (also see Kobulnicky \& Johnson 1999 and Johnson \& Kobulnicky 2003). Very long baseline interferometry (VLBI) observations constrain the size of the 


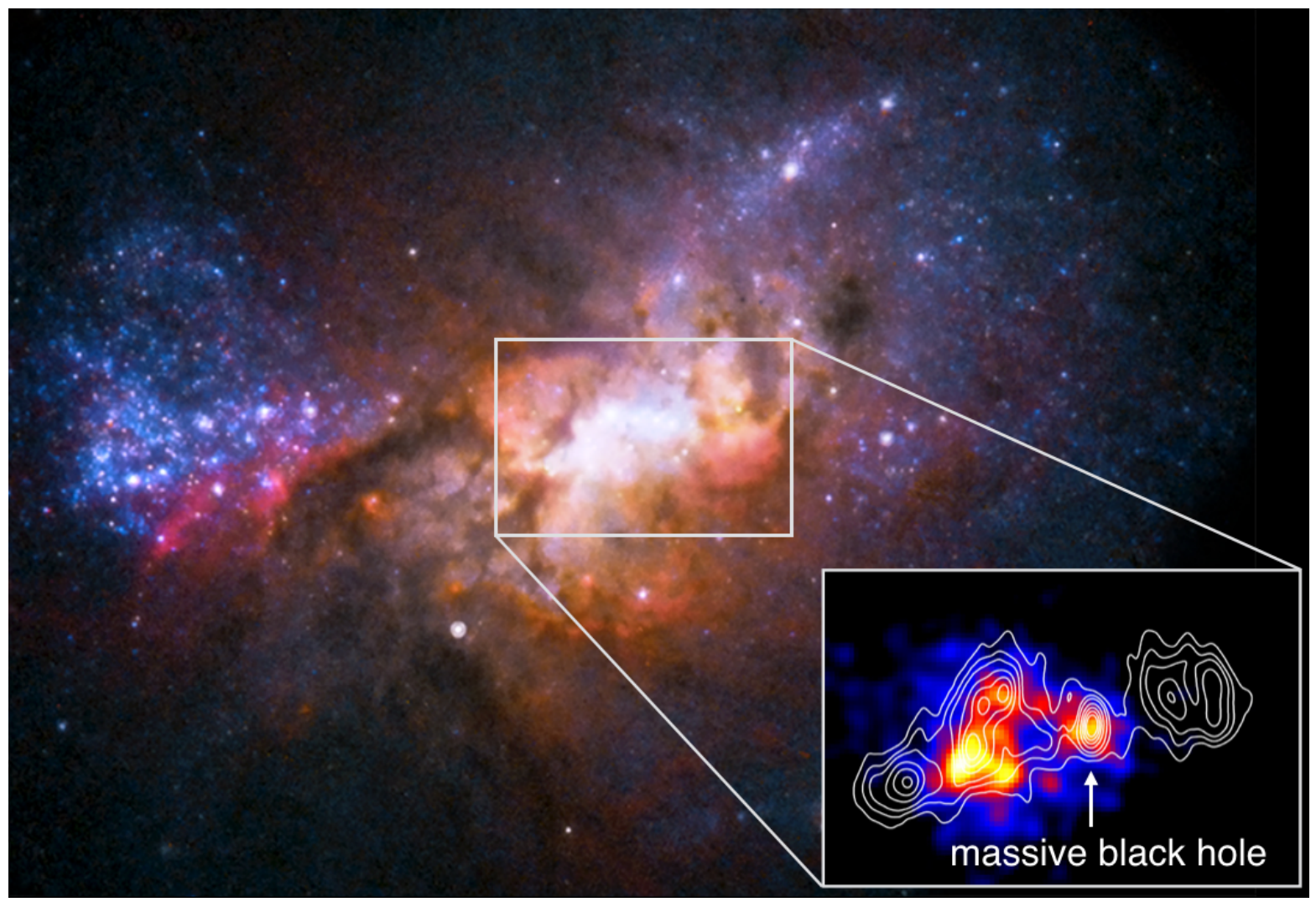

Figure 1. HST image of Henize 2-10. The inset shows our new $160 \mathrm{ks}$ Chandra observation with VLA radio contours from Reines et al. (2011) and has dimensions $6^{\prime \prime} \times 4^{\prime \prime}(\sim 265 \mathrm{pc} \times 175 \mathrm{pc})$.

nuclear radio emission to $<1 \mathrm{pc} \times 3 \mathrm{pc}$ and the high brightness temperature of the radio core confirms a nonthermal origin (Reines \& Deller 2012).

Chandra X-ray Observatory observations of Henize 210 taken in 2001 show point-like hard X-ray emission that has previously been associated with the nuclear radio source (Ott et al. 2005; Kobulnicky \& Martin 2010, Reines et al. 2011). Here we demonstrate that this emission is dominated by a source that is highly variable (also see Whalen et al. 2015) and not in fact co-spatial with the radio source. Our new deep Chandra observations expose a different, previously unidentified X-ray counterpart to the nuclear radio source (Figure 1), for which we examine the $\mathrm{X}$-ray spectrum and light curve.

\section{OBSERVATIONS AND DATA REDUCTION}

We obtained new Chandra observations of Henize 2-10 in February 2015. The total exposure time of $\sim 200 \mathrm{ks}$ was broken up into two observations of $159066 \mathrm{~s}$ and 37577 s beginning on February 5 and 16 (PI: Reines, ObsIDs 16068 and 16069). These observations were taken in VFAINT mode. We also retrieved the archival observation taken on 2001 March 23 (PI: Martin, ObsID 2075), which was taken in FAINT mode with an exposure time of $19755 \mathrm{~s}$. In all three observations, the galaxy was placed on the S3 chip of the Advanced CCD Imaging Spectrometer (ACIS) detector. The data were reduced and reprocessed with CIAO version 4.6 (Fruscione et al. 2006 ) utilizing CALDB version 4.6.3. To improve the image quality, the data were reprocessed with the EDSER algorithm enabled (Li et al. 2004) and subsequently re- binned to $1 / 8$ th the native ACIS pixel size before convolving with a FWHM $=0.25^{\prime \prime}$ Gaussian. Our spectral and variability analysis is performed on the event files.

To improve the astrometry, we co-aligned the three Chandra observations and then tied the corrected images to the absolute reference frame defined by our radio observations, which is accurate to $\lesssim 0$.' 1 (Reines et al. 2011, Reines \& Deller 2012). We first averaged the coordinates of a bright point source common to all three Chandra observations, yet outside the vicinity of the nuclear region. This reference source is located $\sim 99^{\prime \prime} .5$ east of the nuclear radio source and has a corrected (mean) position of $\mathrm{RA}=8: 36: 15.83, \mathrm{DEC}=-26: 24: 34.1$ (Figure 2). We then registered the three Chandra observations by determining the relative offset between this mean position and the position of the reference source in an individual observation. The required (RA, DEC) shifts in arcseconds are $(0.17 \mathrm{~W}, 0$.'13 N), (0..08 E, 0.'00 S), and (0..08 E, $0.13 \mathrm{~S}$ ) for the $20 \mathrm{ks}, 160 \mathrm{ks}$, and $40 \mathrm{ks}$ observations, respectively. There is no evidence for significant rotation between the different observations. A comparison between our deep $160 \mathrm{ks}$ observation and the VLA contours from Reines et al. (2011) indicates a close match between bright regions of X-ray and radio emission from recent star formation (inset Figure 1), strongly suggesting the absolute astrometry of our corrected Chandra observations is accurate and requires no additional shift. The absolute astrometric uncertainties of the final Chan$d r a$ positions are estimated to be 0.15 in RA and 0 ". 13 in DEC using the standard deviation of the three individual uncorrected measurements of the reference source. 

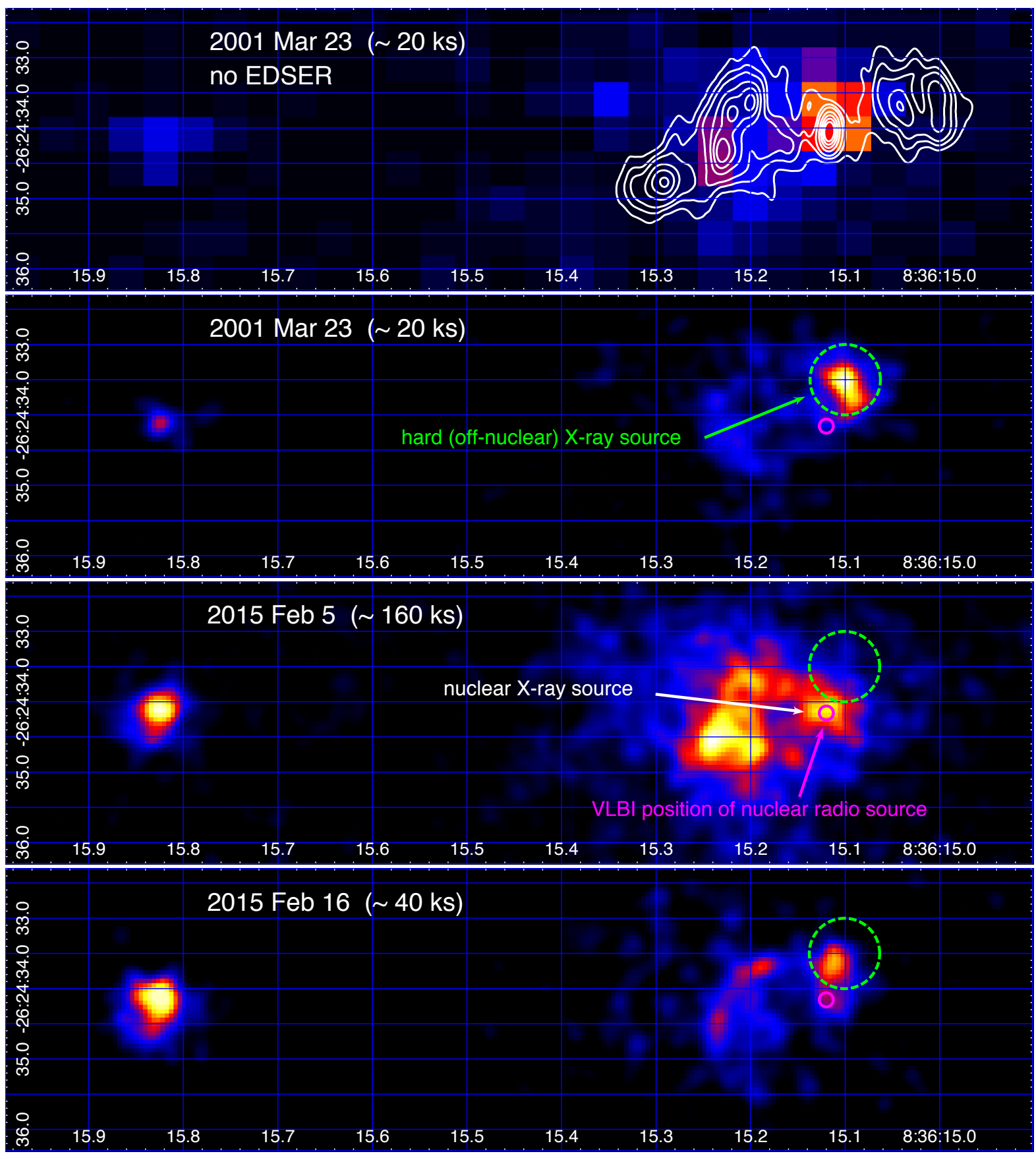

Figure 2. Chandra observations showing the central region of Henize 2-10. All images are in the 0.3-7 keV energy range but not on the same count rate scale. The top panel shows the observation from 2001 used by Reines et al. (2011) without the EDSER algorithm enabled. VLA contours from Reines et al. (2011) are overlaid to show the seeming match between the bright hard X-ray source and the nuclear radio source. The bottom three panels show all observations using the EDSER algorithm. The VLBI position and absolute positional uncertainty of the nuclear radio source from Reines \& Deller (2012) is indicated by a magenta circle with $r=0$. .' 1 . The bright hard X-ray source previously identified in the 2001 observation (also seen in the $40 \mathrm{ks}$ observation from 2015) is indicated by a green circle $\left(r=00^{\prime \prime} 5\right.$ ) and is clearly offset from the nuclear radio source. A newly revealed X-ray source visible in the 160 ks observation from 2015 is co-spatial with the nuclear radio source.

\section{ANALYSIS AND RESULTS}

3.1. The Newly-Identifed Nuclear X-ray Source

Our primary goal in this Letter is to examine the nuclear X-ray source in Henize 2-10. From Figures 1 and 2, we can now see that a previously unidentified $\mathrm{X}$-ray source is spatially coincident with the central radio source, hereafter referred to as the nuclear X-ray source (visible in the $160 \mathrm{ks}$ image at $\mathrm{RA}=8: 36: 15.12$, $\mathrm{DEC}=-26: 24: 34.1)$, and that the bright X-ray emission identified in the 2001 observation (Ott et al. 2005: Kobulnicky \& Martin 2010, Reines et al. 2011. Whalen et al. 2015) is dominated by an X-ray source $(\mathrm{RA}=8: 36: 15.10, \mathrm{DEC}=-26: 24: 33.5)$ distinct from the nuclear radio source. The spatial offset between the 

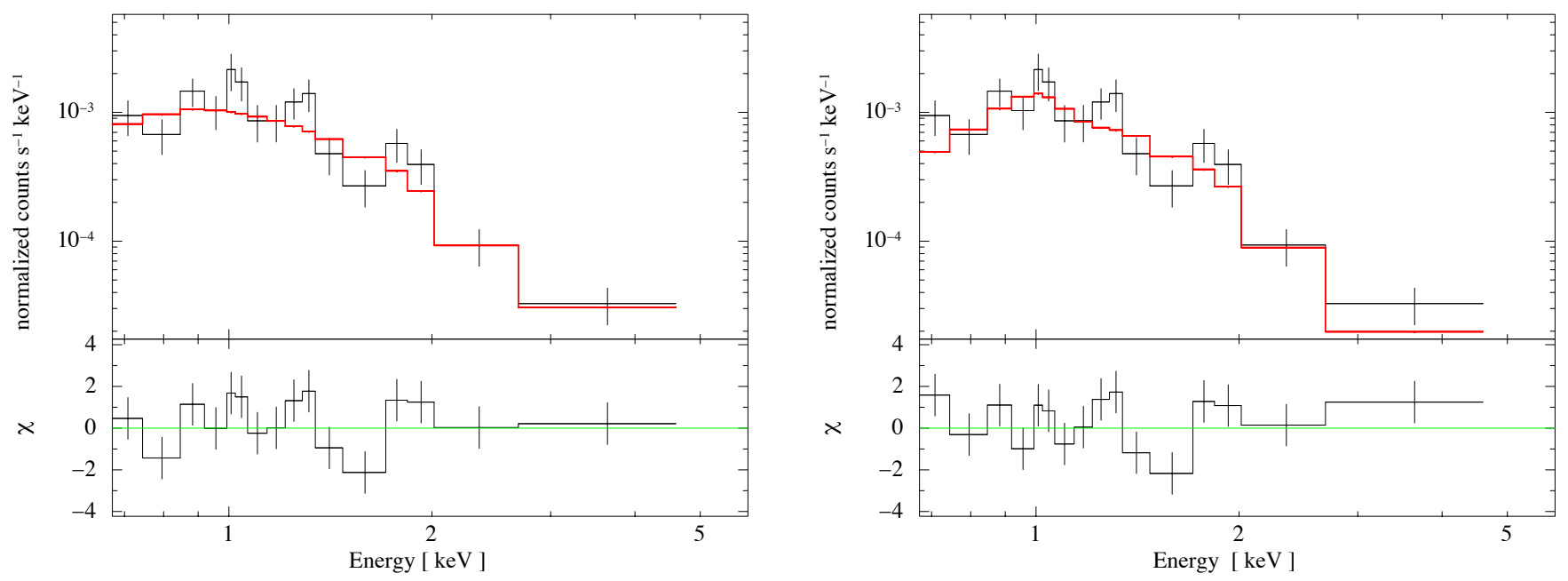

Figure 3. X-ray spectrum of the nuclear source. Best-fit power law model (left) and best-fit thermal plasma model (right), with residuals shown beneath.

bright off-nuclear X-ray source in the subpixel image from 2001 and the VLBI position is $\sim 0 . \prime 7(\sim 30 \mathrm{pc}$ projected). This offset is significantly larger than our astrometric uncertainties, and thus our conclusion that the hard X-ray source is distinct from the nuclear radio source is secure. This source is also highly variable it dominates the emission in 2001 , is essentially absent in our new $160 \mathrm{ks}$ observation, and returns in the $40 \mathrm{ks}$ observation taken only 9 days later.

The spectrum of the bright, off-nuclear variable source dominating the 2001 observation is poorly constrained, but consistent with a highly absorbed power-law $\left(N_{H} \sim\right.$ $\left.7 \times 10^{22} \mathrm{~cm}^{-2}, \Gamma=1.8\right)$. Assuming a common spectral shape in each of the three observations, we measure unabsorbed $0.3-10.0 \mathrm{keV}$ fluxes of $\left(5.09_{-1.24}^{+1.68}, 0.17_{-0.07}^{+0.09}\right.$, and $\left.1.28_{-0.38}^{+0.47}\right) \times 10^{-13} \mathrm{erg} \mathrm{s}^{-1} \mathrm{~cm}^{-2}$ respectively $(90 \%$ confidence intervals), i.e., variability of a factor of $\sim 30$. The large-amplitude variability (also see Whalen et al. 2015) and the large luminosity in the 2001 observation $\left(\geq 10^{39} \mathrm{erg} \mathrm{s}^{-1}\right)$ suggests it is an X-ray binary (XRB) containing a stellar-mass $\mathrm{BH}$ primary.

\subsection{X-ray Spectrum of the Nucleus}

The fortuitous disappearance of the highly variable offnuclear X-ray source in our $160 \mathrm{ks}$ observation enables us to extract a relatively clean X-ray spectrum of the nuclear source. We extracted the spectrum in the 0.3$7.0 \mathrm{keV}$ range from a circular aperture with a radius of 0.5 , correcting for the small aperture. The background was estimated from a source-free annular region extending from 20-25" centered on the nuclear source. We note that the external background contribution is negligible at the position of the nucleus; however, we cannot reliably separate the point source from any local background within the source extraction region. We obtained a total of 183 net counts. The spectrum was grouped to have $\mathrm{SNR}=3$ per bin, and spectral fits to the background subtracted spectrum were carried out within XSPEC 12.8.2q (Arnaud 1996) using the chi statistic and standard Gaussian weighting. Galactic foreground absorption was held fixed at $N_{\mathrm{H}, \mathrm{Gal}}=9.1 \times 10^{20} \mathrm{~cm}^{-2}$ (Kalberla et al. 2005) and the internal absorption was found to be negligible. We used the phabs absorption model, with abundances and cross-sections adopted from Asplund et al. (2009) and Balucinska-Church \& McCammon (1992), respectively.

The source spectrum is well characterized by either an absorbed power-law model with photon index $\Gamma \sim 2.9$, or a thermal plasma model with $k T \sim 1.1 \mathrm{keV}$ (Figure 3. Table 1). Both models give a consistent intrinsic luminosity of $L_{0.3-10 \mathrm{keV}} \sim 10^{38} \mathrm{erg} \mathrm{s}^{-1}$.

\subsection{X-ray Light Curve of the Nucleus}

We also examine the temporal behavior of the nuclear X-ray emission during the $160 \mathrm{ks}$ observation. Utilizing the same extraction regions as used for the spectral analysis, a background subtracted light curve was created using DMEXTRACT. As the count rate is low, the light curve was extracted at $5 \mathrm{ks}$ resolution and Gehrels errors are assumed (Gehrels 1986).

From Figure 4, we can see that the light curve exhibits clear variability (a factor of $\sim 2 \times$ ) and the $\mathrm{X}$ ray emission appears to oscillate within the $160 \mathrm{ks}$ exposure. A model consisting of a constant plus a sine wave provides an excellent characterization of the light curve $\left(\chi^{2} / \nu=9.2 / 29\right)$ and reveals a best fit period of $P=33.5 \pm 2.6 \mathrm{ks}(90 \%$ confidence level $)$. In the right panel of Figure 4, we plot the resulting light curve when folded on the detected period of $P=33.5 \mathrm{ks}(9.3 \mathrm{hrs})$. The amplitude of the sine model is $(4.71 \pm 1.22) \times 10^{-4}$ counts $\mathrm{sec}^{-1}$, and thus is measured to $3.9 \sigma$.

If instead the light curve is modeled as a constant, we obtain a best fit of $\chi^{2} / \nu=18.4 / 32$. An F-test was used to determine that the sine model is a superior description of the data at the $99.9866 \%$ confidence level $(3.8 \sigma)$. This simple statistical test is dependent on the binning of the light curve, e.g., a binning of $3 / 10 \mathrm{ks}$ resolution favors a sine wave over a constant model at the $95.7 \% / 99.3 \%$ level with $P=33.4 \pm 2.2 \mathrm{ks}, 33.8 \pm 1.9 \mathrm{ks}$ respectively. However, the best fit period is robustly determined irrespective of the actual binning. 
Table 1

Spectral Fits to the Nuclear X-ray Source in Henize 2-10

\begin{tabular}{|c|c|}
\hline \multicolumn{2}{|l|}{ Power-Law Model } \\
\hline$\chi^{2} /$ dof & $22.40 / 14$ \\
\hline$\ldots \ldots \ldots \ldots \ldots \ldots \ldots \ldots \ldots$ & $2.93_{-0.33}^{+0.36}$ \\
\hline Normalization $\left(10^{-6}\right.$ photons $\mathrm{cm}^{-2} \mathrm{~s}^{-1} \mathrm{keV}^{-1}$ at $\left.1 \mathrm{keV}\right)$ & $4.30 \pm 0.66$ \\
\hline$F_{0.3-10 \mathrm{keV}}\left(10^{-14} \mathrm{erg} \mathrm{s}^{-1} \mathrm{~cm}^{-2}\right)$, absorbed $\ldots \ldots \ldots \ldots$ & $1.30_{-0.17}^{+0.22}$ \\
\hline $\log L_{0.3-10 \mathrm{keV}}\left(\mathrm{erg} \mathrm{s}^{-1}\right)$, unabsorbed $\ldots \ldots$ & $38.10_{-0.06}^{+0.07}$ \\
\hline
\end{tabular}

\begin{tabular}{|c|c|}
\hline \multicolumn{2}{|l|}{ Thermal Plasma Model (APEC) } \\
\hline$\chi^{2} /$ dof & $22.64 / 13$ \\
\hline$k T(\mathrm{keV})$ & $1.06_{-0.19}^{+0.27}$ \\
\hline $\mathrm{Z}\left(Z_{\odot}\right) \ldots$ & $0.06_{-0.06}^{+0.16}$ \\
\hline Normalization $\left(\frac{10^{-14}}{4 \pi\left\{D_{A}(1+z)\right\}^{2} \int n_{e} n_{H} d V}\right) \ldots$ & $1.97_{-0.73}^{+0.87}$ \\
\hline $\begin{array}{l}F_{0.3-10 \mathrm{keV}}\left(10^{-14} \mathrm{erg} \mathrm{s}^{-1} \mathrm{~cm}^{-2}\right) \text {, absorbed } \ldots \\
\log L_{0.3-10 \mathrm{keV}}\left(\mathrm{erg} \mathrm{s}^{-1}\right) \text {, unabsorbed } \ldots \ldots \ldots\end{array}$ & $\begin{array}{r}0.89_{-0.28}^{+0.12} \\
37.94_{-0.16}^{+0.05}\end{array}$ \\
\hline
\end{tabular}

\footnotetext{
Note. - We adopt $N_{\mathrm{H}, \mathrm{Gal}}=9.1 \times 10^{20} \mathrm{~cm}^{-2}$ due to the Milky Way, and $N_{\mathrm{H}}=1 \times 10^{20} \mathrm{~cm}^{-2}$ intrinsic to Henize 2-10. The errors represent the $90 \%$ confidence interval for one interesting parameter determined using the error command in XSPEC.
}

The power-spectrum also hints at the presence of a periodic signal. Utilizing the entire observation at the native temporal resolution $(\Delta \mathrm{t}=3.14104 \mathrm{~s})$ reveals the presence of a low significance peak $(\lesssim 2 \sigma)$ at a frequency $f \sim 3 \times 10^{-5} \mathrm{~Hz}(P \sim 33 \mathrm{ks})$ on top of a white noise background. If the signal is in fact periodic, the detected peak in the power-spectrum is likely of low statistical significance due to the small number of cycles sampled in our 160 ks observation $(\lesssim 5)$. However, we cannot rule out the possibility that the seemingly periodic signal could be produced by stochastic variability, which can sometimes mimic intervals of periodicity (e.g., "red noise," Vaughan \& Uttley 2006, Vaughan et al. 2016).

The oscillating signal is uniquely coincident with the nuclear source as identified in our sub-pixel spatial analysis. We have examined light curves from the bright region $2^{\prime \prime}$ to the east and find no evidence for any significant periodic signal at this location only 3 ACIS-S pixels from the nucleus. Likewise, analysis of the light curves of the remaining point sources on the ACIS-S3 detector reveals no evidence for periodicity. Neither do we observe significant background flaring or the presence of periodic variability in the background signal on the ACIS-S3 detector during this observation. A period of $\sim 33 \mathrm{ks}$ should also not be due to aspect dithering.

\section{DISCUSSION}

We have shown that a previously unidentified Chandra $\mathrm{X}$-ray point source is spatially coincident $\left(\lesssim 0^{\prime \prime} \cdot 1, \lesssim 5 \mathrm{pc}\right.$ projected) with the non-thermal compact nuclear radio source in Henize 2-10 (Reines \& Deller 2012). Using three separate Chandra observations and improved image processing, we have determined that the bright hard X-ray source that dominated the original 2001 observation (Ott et al. 2005, Kobulnicky \& Martin 2010, Reines et al. 2011, Whalen et al. 2015) is distinct and spatially offset from the nuclear radio source. The previously detected hard $\mathrm{X}$-ray source is highly variable with properties expected for a stellar-mass BH XRB. Fortunately, this source was absent in our new 160 ks observation, which enabled us to extract and analyze a clean spectrum and light curve of the newly revealed nuclear X-ray source.

Our new results from Chandra support a massive BH origin for the nuclear source in Henize 2-10 (Reines et al. 2011). Given a recent estimate for the total stellar mass of Henize 2-10 $\left(M_{\star} \sim 10^{10} M_{\odot} ;\right.$ Nguyen et al. 2014), we expect a nuclear $\mathrm{BH}$ with a mass ${ }^{2}$ of $M_{\mathrm{BH}} \sim 3 \times 10^{6} M_{\odot}$ (Reines \& Volonteri 2015), although the uncertainty is at least a factor of a few. The luminosity of the nuclear

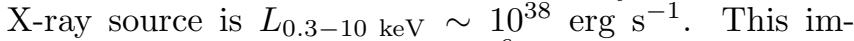
plies an Eddington ratio of $\sim 10^{-6}$, assuming an X-ray to bolometric correction of $\sim 10$ (e.g., Vasudevan \& Fabian 2009). The X-ray spectrum is soft and can be well-fit by either a thermal plasma model with $k T \sim 1.1 \mathrm{keV}$ or a power-law model with $\Gamma \sim 2.9$, similar to Sagittarius $A^{*}$ at the center of the Milky Way (Baganoff et al. 2003) and other massive BHs accreting at very low Eddington ratios (e.g., Constantin et al. 2009). The presence of a spatially coincident non-thermal radio source, with a physical size of $\lesssim 1$ pc $\times 3$ pc $($ Reines \& Deller 2012), also strongly suggests a massive BH. Furthermore, the nuclear source in Henize 2-10 falls along the correlation between nuclear radio and X-ray luminosity for low-luminosity radio galaxies (within the $1 \sigma$ scatter) found by Panessa et al. (2007).

Our temporal analysis of the nuclear X-ray emission reveals clear variability and a potential $\sim 9$-hr periodicity.

2 Coincidentally, this is approximately the same mass estimated by Reines et al. (2011) using the BH fundamental plane (Merloni et al. 2003) and the luminosity of the bright variable source that dominated the 2001 Chandra observation. Using the luminosity of the nuclear source in our new observation $\left(L_{\mathrm{X}} \sim 10^{38} \mathrm{erg} \mathrm{s}^{-1}\right.$, when the transient source was "off") and a $5 \mathrm{GHz}$ radio luminosity of $L_{\mathrm{R}} \sim 4 \times 10^{35} \mathrm{erg} \mathrm{s}^{-1}$ (Reines \& Deller 2012), the fundamental plane gives $\log \left(M_{\mathrm{BH}} / M_{\odot}\right) \sim 7 \pm 1$, consistent with the estimate based on the scaling between $M_{\mathrm{BH}}$ and $M_{\star}$ from Reines \& Volonteri (2015). 

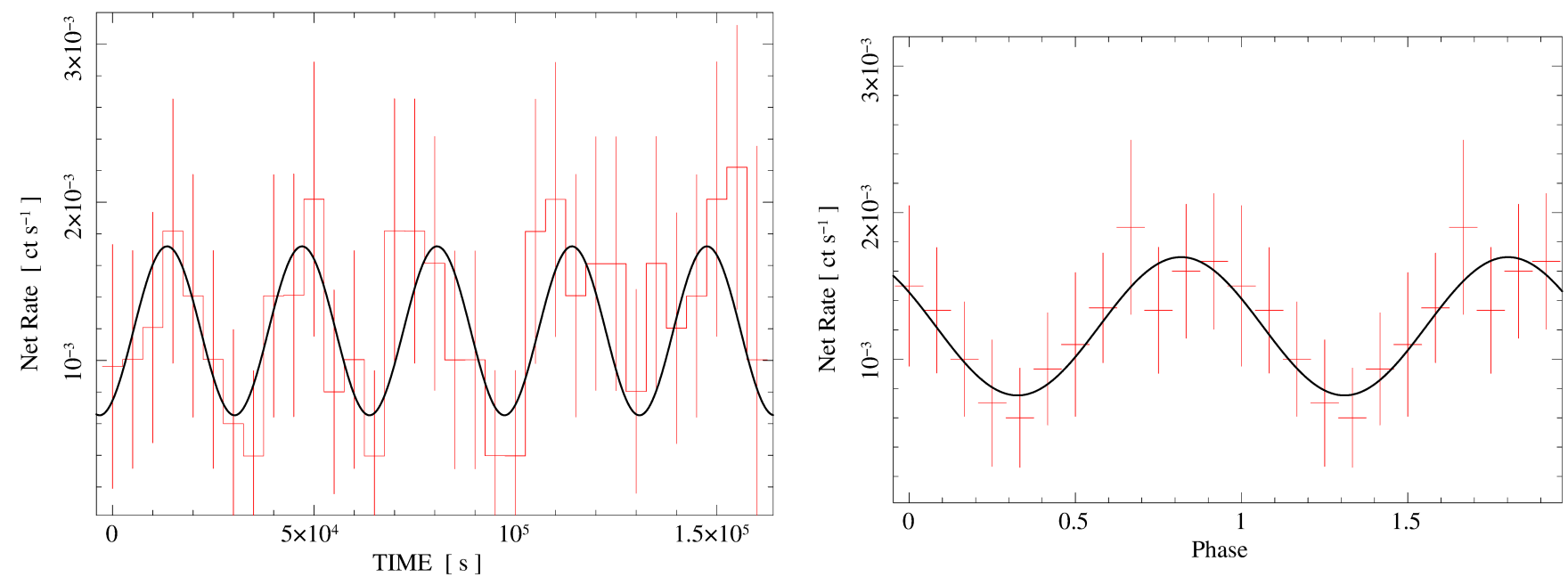

Figure 4. Oscillating X-ray emission from the nuclear source. Left: Light curve binned at 5 ks resolution with the best fit sine model overplotted. Right: Light curve folded on the best fit period of $P=33.5 \mathrm{ks}$ and rebinned to 12 bins per cycle. Two cycles are displayed for clarity.

The oscillatory signal is apparent by eye and significant when fitting the light curve. However, the X-ray periodicity is not yet significant when considering the power spectrum, and at present we cannot distinguish between a true periodic signal and random fluctuations in brightness mimicking a periodic signal during our $160 \mathrm{ks}$ observation (e.g., red noise, see Vaughan \& Uttley 2006; Vaughan et al. 2016). Nevertheless, we discuss possible origins for a $\sim 9$-hr X-ray periodicity below.

The most likely origin is a low frequency quasi-periodic oscillation (LFQPO; Remillard \& McClintock 2006). QPOs are generally thought to arise from instabilities in the accretion flow (e.g., Tagger \& Pellat 1999) or geometric oscillations (e.g., Chakrabarti \& Molteni 1993). It has been proposed that LFQPOs may be due to the orbital precession of non-equitorial particles in the dragged spacetime around a spinning BH (i.e., Lense-Thirring precession; Stella \& Vietri 1998). Given the timescale of the apparent periodicity and the low luminosity, current observational constraints on such variability are limited, with only a single claimed detection of a LFQPO from a massive $\mathrm{BH}$ that is accreting at a relatively high rate $\left(\sim 0.1 L_{\text {Edd }}\right.$, Lin et al. 2013).

We also considered a high frequency QPO (HFQPO) as an origin for the $\mathrm{X}$-ray periodicity, however the observed luminosity and frequency argue against this. The known stellar and massive BH QPO detections that are consistent with a HFQPO origin are all from sources known to be accreting at close to the Eddington limit (e.g., Remillard \& McClintock 2006; Gierliński et al. 2008, Alston et al. 2014: Reis et al. 2012: Pan et al. 2016: Alston et al. 2015). HFQPOs are thought to originate in the inner regions of the accretion flow in the immediate vicinity of the BH. The observed relation between QPO frequency and BH mass (e.g., Remillard \& McClintock 2006; Zhou et al. 2015; Pan et al. 2016) would predict a mass of $\sim 10^{8} M_{\odot}$ for a frequency of $f \sim 3 \times 10^{-5} \mathrm{~Hz}$, more than an order of magnitude greater than the expected mass of the $\mathrm{BH}$ in Henize 2-10.

A final intriguing possibility is orbital variability related to a massive BH binary, where at least one of the $\mathrm{BHs}$ is actively accreting, albeit at a low level (Roedig et al. 2012, 2014, Sesana 2013). In this case the periodicity would correspond to the serendipitous electromagnetic discovery of a massive BH binary with $\lesssim 5$ years until merger, for an assumed total system mass of $\sim 10^{6} M_{\odot}$ (Sesana 2013). Although there is evidence that Henize 2-10 has experienced a merger in the recent past (Kobulnicky et al. 1995), and thus the presence of two massive $\mathrm{BHs}$ is possible, we regard this possibility as unlikely given the extremely short timescale until coalescence implied by the observed frequency.

While we consider a weakly accreting massive $\mathrm{BH}$ the most likely origin for the nuclear X-ray/radio source in Henize 2-10, we nonetheless revisit alternative explanations including a stellar-mass XRB and/or a young supernova remnant (SNR; also see the Supplementary Information in Reines et al. 2011 as well as the discussion in Reines \& Deller 2012). Neither an XRB nor a SNR alone can account for both the X-ray and radio properties of the nuclear source. The radio emission is simply too luminous to be produced by an XRB, especially one in the soft thermal state (Fender et al. 2009) as would be indicated by the observed spectrum, and the X-ray variability is incompatible with a SNR. Our constraints on the positions of the nuclear radio and X-ray sources, however, strongly suggest a common/related source. In principle, we can imagine a scenario in which the X-rays originate from an accreting stellar-mass XRB residing within the radio-emitting remnant of the supernova that created the compact object. Given the size and luminosity of the radio source, a SNR would likely be only decades old (Reines \& Deller 2012, Fenech et al. 2010) and therefore the nuclear X-ray source would be the new record-holder for the youngest XRB known by a wide margin (e.g., Circinus X-1 has an age $t<4600$ yr; Heinz et al. 2013). While we cannot definitively rule out this scenario, we consider it somewhat contrived. Moreover, the radio/X-ray source is at the center of the galaxy (the natural place for a massive $\mathrm{BH}$ ) and there is no star cluster or recent star formation at the location of the source as would be expected for a young XRB/SNR origin (Reines et al. 2011).

Finally, our study demonstrates the value of X-ray 
imaging at sub-arcsecond scales and emphasizes the need for a high-resolution next generation X-ray observatory. Our detection of the first potential LFQPO from a lowluminosity massive $\mathrm{BH}$ would not have been possible with any existing X-ray observatory other than Chandra. Future studies of this class of objects (e.g., with $A T H E N A$ and the X-ray Surveyor) would open a new window to study low luminosity accretion flows, which are the dominant mode of $\mathrm{BH}$ accretion on cosmological scales.

We thank Richard Plotkin for useful discussions and the anonymous referee for reviewing our work. AER is grateful for support from NASA through Hubble Fellowship grant HST-HF2-51347.001-A awarded by the Space Telescope Science Institute, which is operated by the Association of Universities for Research in Astronomy, Inc., for NASA, under contract NAS 5-26555. Support for this work was also provided by NASA through Chandra Award Number GO4-15098A issued by the Chandra Xray Observatory Center, which is operated by the Smithsonian Astrophysical Observatory for and on behalf of the NASA under contract NAS8-03060. GRS acknowledges support by an NSERC Discovery Grant.

\section{REFERENCES}

Alston, W. N., Markevičiūtè, J., Kara, E., Fabian, A. C., \& Middleton, M. 2014, MNRAS, 445, L16

Alston, W. N., Parker, M. L., Markevičiūtè, J., et al. 2015 MNRAS, 449, 467

Arca-Sedda, M., Capuzzo-Dolcetta, R., Antonini, F., \& Seth, A. 2015, ApJ, 806, 220

Arnaud, K. A. 1996, in Astronomical Society of the Pacific Conference Series, Vol. 101, Astronomical Data Analysis Software and Systems V, ed. G. H. Jacoby \& J. Barnes, 17

Asplund, M., Grevesse, N., Sauval, A. J., \& Scott, P. 2009 ARA\&A, 47, 481

Baganoff, F. K., Maeda, Y., Morris, M., et al. 2003, ApJ, 591, 891

Baldassare, V. F., Reines, A. E., Gallo, E., \& Greene, J. E. 2015, ApJ, 809, L14

Baldassare, V. F., Reines, A. E., Gallo, E., \& Greene, J. E. 2016, arXiv:1609.07148

Balucinska-Church, M., \& McCammon, D. 1992, ApJ, 400, 699

Chakrabarti, S. K., \& Molteni, D. 1993, ApJ, 417, 671

Constantin, A., Green, P., Aldcroft, T., et al. 2009, ApJ, 705, 1336
Fender, R. P., Homan, J., \& Belloni, T. M. 2009, MNRAS, 396, 1370

Fenech, D., Beswick, R., Muxlow, T. W. B., Pedlar, A., \& Argo, M. K. 2010, MNRAS, 408, 607

Fruscione, A., McDowell, J. C., Allen, G. E., et al. 2006, in Society of Photo-Optical Instrumentation Engineers (SPIE) Conference Series, Vol. 6270, Society of Photo-Optical Instrumentation Engineers (SPIE) Conference Series

Gehrels, N. 1986, ApJ, 303, 336

Gierliński, M., Middleton, M., Ward, M., \& Done, C. 2008, Nature, 455, 369

Hainline, K. N., Reines, A. E., Greene, J. E., \& Stern, D. 2016, arXiv: 1609.06721

Heinz, S., Sell, P., Fender, R. P., et al. 2013, ApJ, 779, 171

Johnson, K. E., \& Kobulnicky, H. A. 2003, ApJ, 597, 923

Johnson, K. E., Leitherer, C., Vacca, W. D., \& Conti, P. S. 2000, AJ, 120, 1273

Kalberla, P. M. W., Burton, W. B., Hartmann, D., et al. 2005, A\&A, 440, 775

Kobulnicky, H. A., Dickey, J. M., Sargent, A. I., Hogg, D. E., \& Conti, P. S. 1995, AJ, 110, 116

Kobulnicky, H. A., \& Johnson, K. E. 1999, ApJ, 527, 154

Kobulnicky, H. A., \& Martin, C. L. 2010, ApJ, 718, 724

Lemons, S. M., Reines, A. E., Plotkin, R. M., Gallo, E., \& Greene, J. E. 2015, ApJ, 805, 12

Li, J., Kastner, J. H., Prigozhin, G. Y., et al. 2004, ApJ, 610, 1204

Lin, D., Irwin, J. A., Godet, O., Webb, N. A., \& Barret, D. 2013, ApJ, 776, L10

Merloni, A., Heinz, S., \& di Matteo, T. 2003, MNRAS, 345, 1057

Nguyen, D. D., Seth, A. C., Reines, A. E., et al. 2014, ApJ, 794, 34

Ott, J., Walter, F., \& Brinks, E. 2005, MNRAS, 358, 1423

Pan, H.-W., Yuan, W., Yao, S., et al. 2016, ApJ, 819, L19

Panessa, F., Barcons, X., Bassani, L.. et al. 2007. A\&A, 467, 519

Reines, A., \& Comastri, A. 2016, arXiv:1609.03562

Reines, A. E. \& Deller, A. T 2012, ApJ, 750, L24

Reines, A. E., Greene, J. E., \& Geha, M. 2013, ApJ, 775, 116

Reines, A. E., Plotkin, R. M., Russell, T. D., et al. 2014, ApJ, 787, L30

Reines, A. E., Sivakoff, G. R., Johnson, K. E., \& Brogan, C. L. 2011, Nature, 470, 66

Reines, A. E., \& Volonteri, M. 2015, ApJ, 813, 82

Reis, R. C., Miller, J. M., Reynolds, M. T., et al. 2012, Science, 337,949

Remillard, R. A., \& McClintock, J. E. 2006, ARA\&A, 44, 49

Roedig, C., Krolik, J. H., \& Miller, M. C. 2014, ApJ, 785, 115

Roedig, C., Sesana, A., Dotti, M., et al. 2012, A\&A, 545, A127

Sesana, A. 2013, Classical and Quantum Gravity, 30, 244009

Stella, L., \& Vietri, M. 1998, ApJ, 492, L59

Tagger, M., \& Pellat, R. 1999, A\&A, 349, 1003

Vasudevan, R. V., \& Fabian, A. C. 2009, MNRAS, 392, 1124

Vaughan, S., \& Uttley, P. 2006, Advances in Space Research, 38, 1405

Vaughan, S., Uttley, P., Markowitz, A. G., et al. 2016, MNRAS, 461,3145

Whalen, T. J., Hickox, R. C., Reines, A. E., et al. 2015, ApJ, 806, 37

Zhou, X.-L., Yuan, W., Pan, H.-W., \& Liu, Z. 2015, ApJ, 798, L5 